\title{
Adiabatic superconducting artificial neural network: Basic cells
}

Igor I. Soloviev, Andrey E. Schegolev, Nikolay V. Klenov, Sergey V. Bakurskiy, Mikhail Yu. Kupriyanov, Maxim V. Tereshonok, Anton V. Shadrin, Vasily S. Stolyarov, and Alexander A. Golubov

Citation: Journal of Applied Physics 124, 152113 (2018); doi: 10.1063/1.5042147

View online: https://doi.org/10.1063/1.5042147

View Table of Contents: http://aip.scitation.org/toc/jap/124/15

Published by the American Institute of Physics

\section{Articles you may be interested in}

Theoretical basis of SQUID-based artificial neurons

Journal of Applied Physics 124, 152106 (2018); 10.1063/1.5037718

Chain of magnetic tunnel junctions as a spintronic memristor

Journal of Applied Physics 124, 152116 (2018); 10.1063/1.5042431

Ultra-fast logic devices using artificial "neurons" based on antiferromagnetic pulse generators

Journal of Applied Physics 124, 152115 (2018); 10.1063/1.5042348

Overcoming device unreliability with continuous learning in a population coding based computing system Journal of Applied Physics 124, 152111 (2018); 10.1063/1.5042250

Integrate-and-fire neuron circuit using positive feedback field effect transistor for low power operation Journal of Applied Physics 124, 152107 (2018); 10.1063/1.5031929

Tutorial: Fabrication and three-dimensional integration of nanoscale memristive devices and arrays Journal of Applied Physics 124, 152001 (2018); 10.1063/1.5038109

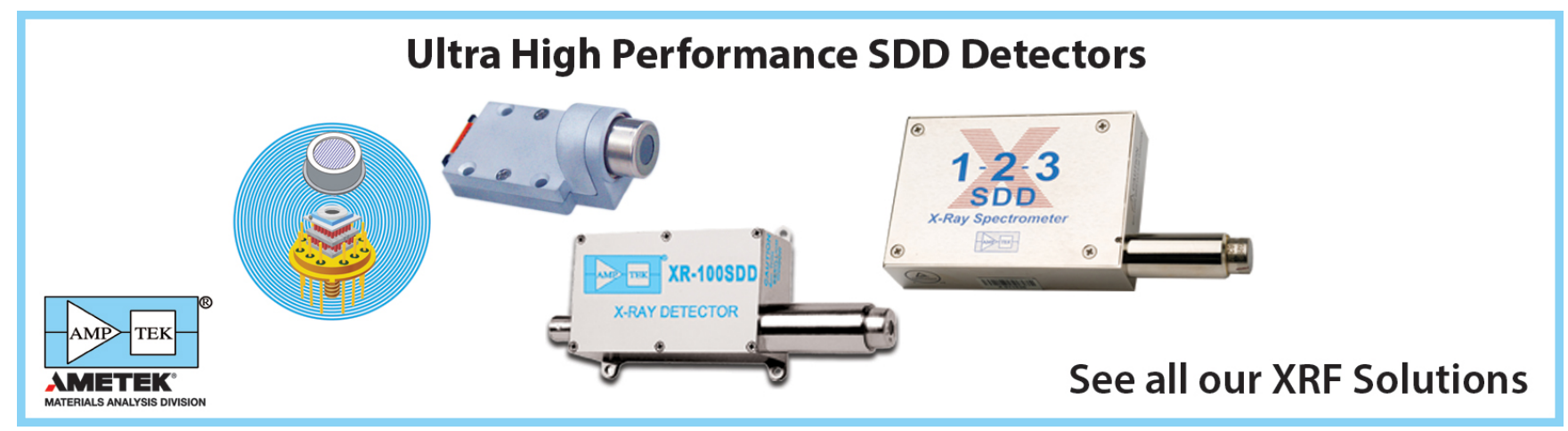




\title{
Adiabatic superconducting artificial neural network: Basic cells
}

\author{
Igor I. Soloviev, ${ }^{1,2,3, a)}$ Andrey E. Schegolev, ${ }^{1,2,4,5}$ Nikolay V. Klenov, ${ }^{1,2,4,5,6}$ \\ Sergey V. Bakurskiy, ${ }^{1,2,3}$ Mikhail Yu. Kupriyanov, ${ }^{1}$ Maxim V. Tereshonok, ${ }^{2,5}$ \\ Anton V. Shadrin, ${ }^{3}$ Vasily S. Stolyarov, ${ }^{3,6,7,8}$ and Alexander A. Golubov ${ }^{3,9}$ \\ ${ }^{1}$ Lomonosov Moscow State University Skobeltsyn Institute of Nuclear Physics, 119991 Moscow, Russia \\ ${ }^{2}$ MIREA—Russian Technological University, 119454 Moscow, Russia \\ ${ }^{3}$ Moscow Institute of Physics and Technology, 141700 Dolgoprudny, Russia \\ ${ }^{4}$ Physics Department, Lomonosov Moscow State University, 119991 Moscow, Russia \\ ${ }^{5}$ Moscow Technical University of Communications and Informatics (MTUCI), 111024 Moscow, Russia \\ ${ }^{6}$ Dukhov Research Institute of Automatics (VNIIA), 127055 Moscow, Russia \\ ${ }^{7}$ Institute of Solid State Physics RAS, 142432 Chernogolovka, Russia \\ ${ }^{8}$ Solid State Physics Department, KFU, 420008 Kazan, Russia \\ ${ }^{9}$ Faculty of Science and Technology and MESA+ Institute of Nanotechnology, 7500 AE Enschede, \\ The Netherlands
}

(Received 30 May 2018; accepted 25 July 2018; published online 26 September 2018)

\begin{abstract}
We consider adiabatic superconducting cells operating as an artificial neuron and synapse of a multilayer perceptron (MLP). Their compact circuits contain just one and two Josephson junctions, respectively. While the signal is represented as magnetic flux, the proposed cells are inherently nonlinear and close-to-linear magnetic flux transformers. The neuron is capable of providing the oneshot calculation of sigmoid and hyperbolic tangent activation functions most commonly used in MLP. The synapse features both positive and negative signal transfer coefficients in the range $\sim(-0.5,0.5)$. We briefly discuss implementation issues and further steps toward the multilayer adiabatic superconducting artificial neural network, which promises to be a compact and the most energy-efficient implementation of MLP. Published by AIP Publishing.

https://doi.org/10.1063/1.5042147
\end{abstract}

\section{INTRODUCTION}

Artificial neural network (ANN) is the key technology in the fast developing area of artificial intelligence. It has been already broadly introduced in our everyday life. Further progress requires an increase in complexity and depth of ANNs. However, modern implementations of the neural networks are commonly based on conventional computer hardware which is not well suited for neuromorphic operation. This leads to excessive power consumption and hardware overhead. Ideal basic elements of ANNs should combine the multiple properties like one-shot calculation of their functions, operation with energy near the thermal noise floor, and nanoscale dimensions.

The most energy efficient computing today can be performed using the superconductor digital technology. ${ }^{1}$ The first ever practical logic gates capable of operating down to and below the Landauer thermal $\operatorname{limit}^{2}$ were realized recently ${ }^{3}$ on the basis of adiabatic superconductor logic. Besides the several attempts to the implementation of the superconducting ANNs proposed since the $1990 \mathrm{~s},{ }^{4-12}$ the idea to adopt the adiabatic logic cells to neuromorphic circuits was presented only recently. ${ }^{13,14}$ In this paper, we consider operation principles of adiabatic superconducting basic cells which comply with the above-mentioned properties for ANN implementation. We focus on a particular multilayer

a)isol@phys.msu.ru perceptron (MLP) because of a wide range of its applicability and well-developed learning algorithms for such a network.

\section{BASIC CELLS}

The basic element of superconducting circuits is the Josephson junction. Its characteristic energy typically lies below aJ level while switching frequency is several hundred GHz. Contrary to semiconductor transistor, the Josephson junction is not fabricated in a substrate but between two superconductor layers deposited on a substrate utilized as a mechanical support. This provides opportunity for superconducting circuits to benefit from 3D topology which can be especially suitable for deep ANNs. The minimal feature size of superconducting circuits is progressively decreased down to nanoscales in recent years. ${ }^{15}$

Another attractive feature of the Josephson junction is its inherently strong nonlinearity. Indeed, the current flowing through the junction, $I$, is commonly related to the superconducting phase difference between the superconducting banks, $\varphi$, as

$$
I=I_{c} \sin \varphi,
$$

where $I_{c}$ is the junction critical current. We show below that this current-phase relation (CPR) having both linear and nonlinear parts is well suited for implementation of superconducting artificial neuron with one-shot calculation of sigmoid or hyperbolic tangent activation functions

$$
\sigma(x)=\frac{1}{1+e^{-x}}
$$


or

$$
\tau(x)=\tanh (x),
$$

utilized in MLP and superconducting synapse enabling signal transfer with both positive and negative coefficients. Unlike most of their predecessors, ${ }^{4-9,11,12}$ both cells are operating in a pure superconducting mode featured by minimal power consumption.

\section{A. Artificial neuron}

One of the simplest superconducting cells is parametric quantron proposed in 1982 for adiabatic operation. ${ }^{16}$ It is the superconducting loop consisted of a Josephson junction and a superconducting inductance. According to the Josephson junction CPR (1), the relation between the input magnetic flux and the Josephson junction phase in its circuit has a simple expression:

$$
\varphi+l \sin \varphi=\phi_{i n},
$$

where we use normalization of current to critical current of the Josephson junction, $I_{c}$, and input magnetic flux $\Phi_{i n}$ to the magnetic flux quantum $\Phi_{0}, \phi_{i n}=2 \pi \Phi_{i n} / \Phi_{0}$, inductance, $\mathrm{L}$, is normalized to characteristic inductance, $l=L / L_{c}$, $L_{c}=\Phi_{0} / 2 \pi I_{c}$, accordingly.

It is seen from (1) and (3) that the current circulating in the loop has a tilted sine dependence on input magnetic flux. The way to transform this dependence close to the desired one $[(2 \mathrm{a})$ or $(2 \mathrm{~b})]$ is the addition of a linear term compensating the sine slope on the initial section (where $\sin \varphi \approx \varphi$ ) in the vicinity of zero input flux, $\phi_{\text {in }} \approx 0$.

This can be done by attaching another superconducting loop with a part of its inductance, $l_{\text {out }}$, being common with the initial circuit [see Fig. 1(a)]. The synthesized cell was named a "sigma-cell" 13 because its transformation of magnetic flux can be very close to sigmoid function. Here, we are interested in a transfer function, $\phi_{\text {out }}\left(\phi_{\text {in }}\right)$, where output magnetic flux, $\phi_{\text {out }}$, is proportional to output current, $\phi_{\text {out }}=l_{\text {out }} i_{\text {out }}$.

The system of equations describing the proposed cell is as follows:

$$
\begin{gathered}
\varphi+l \sin \varphi=\phi_{\text {in }} / 2+l_{\text {out }} i_{\text {out }}, \\
\varphi+l \sin \varphi=\phi_{\text {in }}+l_{a} i_{a},
\end{gathered}
$$

where $l_{a}$ is the attached inductance. The corresponding system implicitly defining the transfer function through dependencies of $\phi_{\text {out }}, \phi_{\text {in }}$ on $\varphi$ has the following form:

$$
\begin{gathered}
\phi_{\text {out }}=l_{\text {out }} \frac{\phi_{\text {in }}-2 l_{a} \sin \varphi}{2\left(l_{a}+l_{\text {out }}\right)}, \\
\phi_{\text {in }}=2\left(\frac{l_{a}+l_{\text {out }}}{l_{a}+2 l_{\text {out }}}\right)\left[\varphi+\left(l+\frac{l_{a} l_{\text {out }}}{l_{a}+l_{\text {out }}}\right) \sin \varphi\right] .
\end{gathered}
$$

Vanishing of the derivative $d \phi_{\text {out }} / d \phi_{\text {in }}$ at $\phi_{\text {in }}=0$ corresponds to the condition:

$$
l_{a}=1+l
$$

a
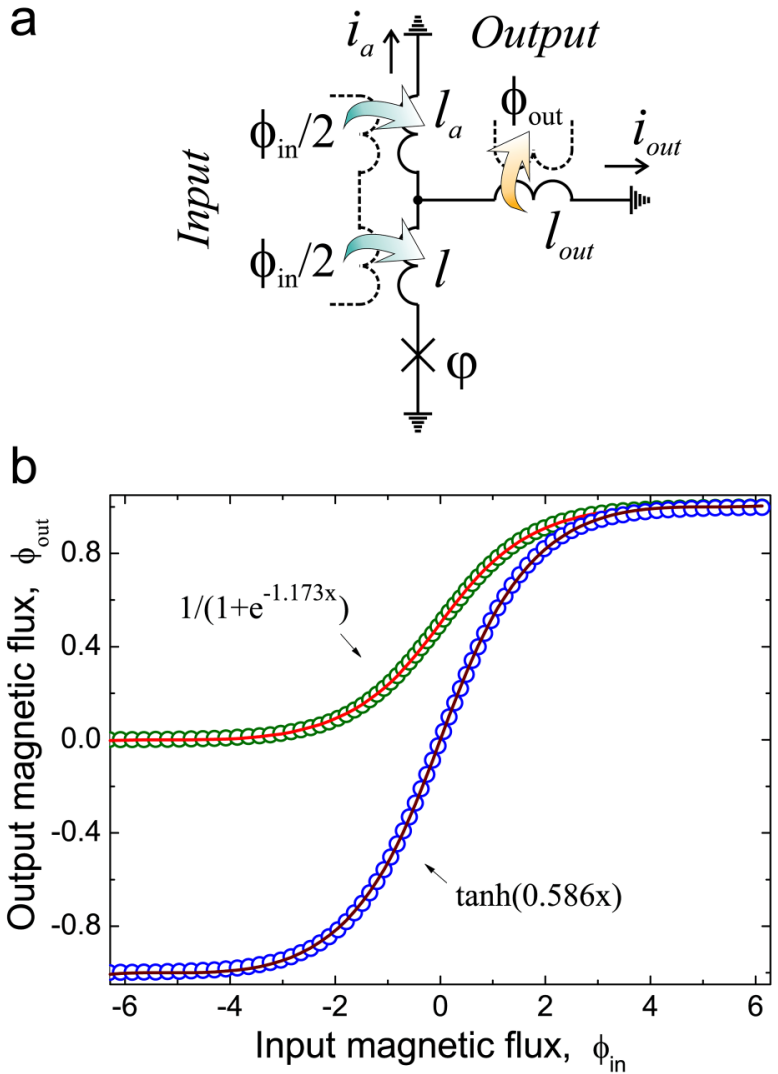

FIG. 1. (a) Scheme of an artificial neuron cell. (b) The cell transfer function (line) fitted to sigmoid and hyperbolic tangent functions (dots). Scaling of the functions (2) is shown in the figure. The transfer function $\phi_{\text {out }}\left(\phi_{i n}\right)$ is normalized by $2 \pi l_{\text {out }} /\left(l_{a}+2 l_{\text {out }}\right)$ and shifted by $-2 \pi\left(l_{a}+l_{\text {out }}\right) /\left(l_{a}+2 l_{\text {out }}\right)$ on the flux axis to fit (2a), and normalized to $\pi l_{\text {out }} /\left(l_{a}+2 l_{\text {out }}\right)$ with no additional shift on flux axis to fit (2b). The optimal values of parameters are $l=0.125, l_{\text {out }}=0.3, l_{a}=1.125$. Consistency of curves in both cases is at the level of $10^{-3}$. Hyperbolic tangent activation function is fitted with $\pi$ shift in the Josephson junction CPR (1).

One can fit (5) to sigmoid function (2a) taking (6) into account with the two fitting parameters: $l, l_{\text {out }}$.

The result of fitting is shown in Fig. 1(b). The found optimal values, $l=0.125, l_{\text {out }}=0.3$, provide conformity of the sigma-cell transfer function with sigmoid one with standard deviation at the level of $10^{-3}$. Sigmoid function (2a) was scaled as $\sigma(1.173 x)$ in our fitting process. The transfer function $\phi_{\text {out }}\left(\phi_{\text {in }}\right)$ (5) was normalized by $2 \pi l_{\text {out }} /\left(l_{a}+2 l_{\text {out }}\right)$ to fit a unit height and shifted by a half period. The latter can be obtained by application of a constant bias flux to the circuit, $\phi_{b}=-2 \pi\left(l_{a}+l_{\text {out }}\right) /\left(l_{a}+2 l_{\text {out }}\right)$.

While sigmoid activation function is commonly used for input data defined in the positive domain, for data defined on the whole numeric axis around zero, it is convenient to use hyperbolic tangent. Application of additional bias flux providing $\pi$ phase shift into the loop containing Josephson junction moves the center of the nonlinear part of the cell transfer function to zero. This allows one to obtain the desired shape of activation function (2b). The $\pi$ phase shift can also be implemented using the $\pi$-Josephson junction ${ }^{17-20}$ with $\pi$ shift of its CPR (1), $I=-I_{c} \sin (\varphi)$, instead of the standard one.

One needs to correspondingly change the sign of the terms containing sine function in (5) to perform the fitting 
procedure. The fitting result is presented in Fig. 1(b). Hyperbolic tangent function was scaled as $\tanh (0.586 x)$ while the transfer function $\phi_{\text {out }}\left(\phi_{\text {in }}\right)$ was normalized by a factor of two lower value than the previous time, $\pi l_{\text {out }} /\left(l_{a}+2 l_{\text {out }}\right)$. With the same values of parameters $l, l_{\text {out }}$, and zero bias flux, we obtained the same conformity of the curves.

\section{B. Artificial synapse}

Synapse modulates the "weight" of a signal arriving at the neuron. In our case, the signal corresponds to magnetic flux and, therefore, synapse can be implemented simply as a transformer of magnetic flux with desired coupling factor. Summation of signals can be provided by connecting the transformers to a single superconducting input loop of the neuron. However, this solution suits for ANN with a certain and unchangeable configuration.

In most cases, a configurable ANN would be preferable. The selected configuration of inter-neuron connections should be maintained during its entire use if the feature space dimensions do not vary. However, the weight values should be configurable if we want to train the ANN on the fly. The best way to meet this requirement is utilization of some nonvolatile memory elements. In superconducting circuits, such an element can be implemented by using the ferromagnetic (F) materials. In particular, introduction of F-layers into the Josephson junction weak link area allows us to modulate its critical current. ${ }^{1,21,22}$ This phenomenon was already proposed for utilization in artificial synapse of superconducting spiking ANN. ${ }^{12}$ In our case of MLP, we can also make use of it.

The synapse scheme presented in Fig. 2(a) is nearly a mirrored scheme of the proposed neuron [Fig. 1(a)]. The only differences are the addition of the second Josephson junction and the possibility to independently modulate critical currents of the magnetic junctions (marked by boxes), e.g., by application of tuning magnetic field.

For MLP, it is required to provide both positive and negative weights of signal. Our synapse is designed according to this requirement. The input current, $i_{i n}$, induced in inductance $l_{i n}$ by input magnetic flux, $\phi_{i n}$, is split toward the two Josephson junctions. Magnitude of currents $i_{1}, i_{2}$ in each branch correspond to critical currents of the junctions, $i_{c 1}, i_{c 2}$, so that the sign of output circulating current, $i_{c i r}=\left(i_{1}-i_{2}\right) / 2$ (and the direction of output magnetic flux, $\phi_{\text {out }}$ ), is determined by their ratio. Maximum inequality of $i_{c 1}, i_{c 2}$ provides maximum output signal, while equal critical currents correspond to zero transfer coefficient.

It is convenient to present the system of equations for the synapse cell in terms of Josephson junctions phase sum, $\varphi_{+}=\left(\varphi_{1}+\varphi_{2}\right) / 2$, and phase difference, $\varphi_{-}=\left(\varphi_{1}-\varphi_{2}\right) / 2$ :

$$
\begin{gathered}
\varphi_{+}+\left(\frac{l}{2}+l_{i n}\right) i_{i n}+\phi_{i n}=0, \\
\varphi_{-}+l i_{c i r}=0 .
\end{gathered}
$$

Furthermore, introducing the sum $\Sigma i_{c}=i_{c 1}+i_{c 2}$ and difference $\Delta i_{c}=i_{c 1}-i_{c 2}$ of the critical currents and taking (1) into
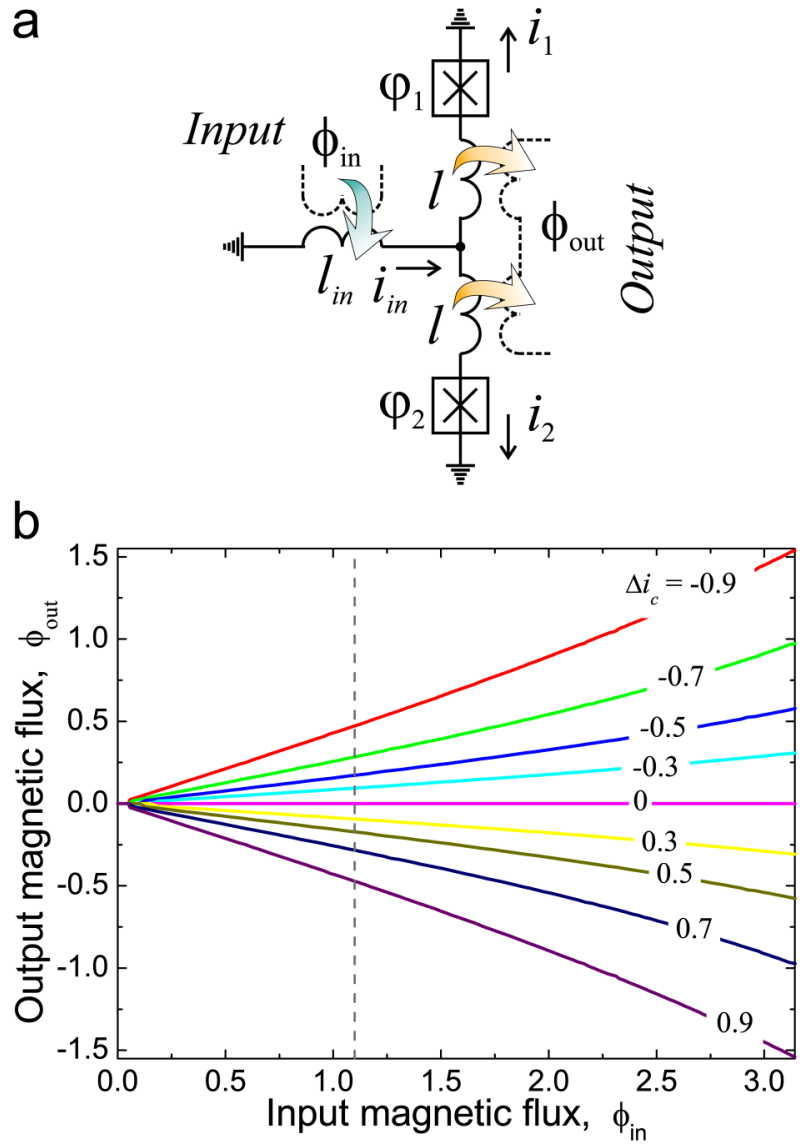

FIG. 2. (a) Scheme of an artificial synapse cell. Magnetic Josephson junctions are marked by boxes. (b) Synapse cell transfer function for the values of parameters: $l_{i n}=2, l=4, \Sigma i_{c}=1$, and $\Delta i_{c}$ as shown in the figure. Vertical dotted line shows the boundary of highly linear range where standard deviation from the linear function is at the level of $10^{-3}$. This range corresponds to maximum output magnetic flux of the optimized neuron cell.

account one can represent (7) in the following form:

$$
\begin{aligned}
\varphi_{+}+ & \left(\frac{l}{2}+l_{i n}\right)\left(\Sigma i_{c} \sin \varphi_{+} \cos \varphi_{-}+\Delta i_{c} \sin \varphi_{-} \cos \varphi_{+}\right) \\
& +\phi_{i n}=0 \\
\varphi_{-}+ & \frac{l}{2}\left(\Sigma i_{c} \sin \varphi_{-} \cos \varphi_{+}+\Delta i_{c} \sin \varphi_{+} \cos \varphi_{-}\right)=0
\end{aligned}
$$

The dependence of the phase difference on the phase sum, $\varphi_{-}\left(\varphi_{+}\right)$, can be obtained ${ }^{23,24}$ from (8b) with corresponding function

$$
\begin{aligned}
f\left(\varphi_{-}, \varphi_{+}\right)= & \varphi_{-} \\
& +\frac{l}{2}\left(\Sigma i_{c} \sin \varphi_{-} \cos \varphi_{+}+\Delta i_{c} \sin \varphi_{+} \cos \varphi_{-}\right),
\end{aligned}
$$

as follows:

$$
\varphi_{-}=\int_{0}^{\pi \operatorname{sgn} \Delta i_{c}} \mathrm{H}\left[-f\left(x, \varphi_{+}\right) \operatorname{sgn} \Delta i_{c}\right] d x,
$$

where $\mathrm{H}(x)$ is the Heaviside step function. Equations (7a), (8a), and (10) implicitly define the cell transfer function 
$\phi_{\text {out }}\left(\phi_{\text {in }}\right)$ through dependencies $\phi_{\text {out }}=2 l i_{\text {cir }}=-2 \varphi_{-}\left(\varphi_{+}\right)$ and $\phi_{i n}\left[\varphi_{-}\left(\varphi_{+}\right), \varphi_{+}\right]$on $\varphi_{+}$. Here, we are interested in the range of the phase sum, $\varphi_{+} \in[0, \pi / 2)$, where the transfer function might be linear.

Figure 2(b) shows synapse cell transfer function for different values of critical currents difference in the range $\Delta i_{c} \in[-0.9,0.9]$. The critical current sum is $\Sigma i_{c}=1$. With the fixed critical currents, the shape of the transfer function is determined by inductances $l_{i n}, l$.

In accordance with (7a), an increase in input inductance $l_{\text {in }}$ increases the amplitude of nonlinearity of the dependence of input current on input flux $i_{i n}\left(\phi_{i n}\right)$ making it more tilted. This is in complete analogy with parametric quantron scheme (3). The slope of the linear part of the transfer function is correspondingly decreased. However, this gives a stretching of this linear part, which is of use for us, and contraction of the nonlinear part.

Increase in inductance $l$ provides the same effect [see (7a)]. At the same time, it increases the nonlinearity of the dependence of output flux on phase sum [see (8b)] which vice versa increases the slope of the linear part though making it less linear. The goal of optimization of the transfer function $\phi_{\text {out }}\left(\phi_{\text {in }}\right)$ is the maximum modulation of its slope alongside with the high linearity among the possibly wider range of input flux.

In our case, the values of inductances were chosen to be $l_{\text {in }}=2, l=4$. With these parameters magnetic flux can be transferred through the synapse with coefficients in the range $\sim(-0.5,0.5)$ depending on the critical currents difference. For maximum output magnetic flux of optimized neuron, $2 \pi l_{\text {out }} /\left(l_{a}+l_{\text {out }}\right) \approx 1.1$, maximum standard deviation of the synapse transfer function from the linear function is at the level of $10^{-3}$. In the whole shown range $[0, \pi]$, it is of an order of magnitude worse.

\section{DISCUSSION}

Both considered cells operate in a pure superconducting regime. Evolution of their states is fully physically reversible. Therefore, they can be operated adiabatically with energy per operation down to the Landauer limit. ${ }^{2}$ For standard working temperature of superconducting circuits, $T=4.2 \mathrm{~K}$, this limit corresponds to the energy, $k_{B} T \ln 2 \approx 4 \times 10^{-23} \mathrm{~J}$ (where $k_{B}$ is the Boltzmann constant). Estimations show that the bit energy can be as low as $10^{-21} \mathrm{~J}$ for adiabatic superconductor logic at clock frequency of $10 \mathrm{GHz} .^{25}$ This is million times less than characteristic energy consumed by a semiconductor transistor. In one hand, taking into account the fact that modern implementation of neuron based on complementarymetal-oxide semiconductor (CMOS) technology requires a few dozens of transistors, the possible gap between power consumption of semiconductor and superconductor ANN is increased by an order. On the other hand, penalty for superconducting circuits cooling is typically several hundred W/W that cancels out the two to three orders of supremacy. Nevertheless, the proposed adiabatic superconducting ANN can be up to $10^{4}-10^{5}$ times more energy efficient than its semiconductor counterparts.
One should note some peculiarities of the proposed concept. First of all, there is no power supply in these circuits and so the signal vanishes. Therefore, there is a need for a flux amplifier which can be implemented on a base of some standard adiabatic cell like adiabatic quantum flux parametron (AQFP). ${ }^{1,26}$ However, such aspects as the linearity of amplification, the distance of signal propagation without amplification, and related issues of achievable fan-in and fan-out should be additionally considered.

Another feature is the periodicity of sigma-cell based neuron transfer function. Corresponding issues can be mitigated by a signal normalization.

Along with the use of standard superconducting integrated circuits fabrication process, the proposed cells require utilization of magnetic Josephson junctions which are relatively new to superconducting technology. Nevertheless, modern developments of cryogenic magnetic memory ${ }^{1,27}$ and superconducting logic circuits with controlled functionality ${ }^{28,29}$ promise their fast introduction.

In particular case of the proposed synapse, one could benefit from implementation of the magnetic Josephson junction controlled by direction of magnetic field, like the Josephson magnetic rotary valve ${ }^{30}$ with heterogeneous area of weak link. Such a valve is featured by high critical current for a certain direction of its F-layer magnetization and low critical current for the direction rotated by $90^{\circ}$. Two such junctions in close proximity to each other with mutual rotation on $90^{\circ}$ relative to their axes directed along the boundary of inhomogeneity allow one to obtain high critical current for one junction and low critical current for another one with the same direction of magnetizations of their F-layers. In this case, rotation of their magnetizations leads to a corresponding decrease and increase of Josephson junction's critical currents which means modulation of synapse weight, according to Fig. 2. Utilization of the rotary valve reduces the number of control lines required to program the magnetic Josephson junctions by half. However, their total number, which is twice the number of synapses, remains huge for practical ANNs. Therefore, the effective synapse control is another urgent task on the way to multilayer adiabatic superconducting ANN.

\section{CONCLUSION}

In this paper, we considered operation principles of adiabatic superconducting basic cells for implementation of multilayer perceptron. These are artificial neuron and synapse which are nonlinear and close-to-linear superconducting transformers of magnetic flux, respectively. Both cells are capable of operation in the adiabatic regime featured by ultra-low power consumption at the level of 4 to 5 orders of magnitude less than that of their modern semiconductor counterparts (including cooling power penalty). The proposed neuron cell contains just a single Josephson junction. The neuron provides one-shot calculation of either sigmoid or hyperbolic tangent activation function. The certain type of this function is determined by the type of utilized Josephson junction and can also be switched on the fly by application of magnetic flux. The synapse is implemented with two magnetic Josephson 
junctions with controllable critical currents. It provides both positive and negative signal transfer coefficients in the range $\sim(-0.5,0.5)$. The presented concept of adiabatic superconducting neuromorphic circuits promises to be a compact and the most energy efficient solution for the artificial neural network of considered type.

\section{ACKNOWLEDGMENTS}

This work was supported by Grant No. 17-12-01079 of the Russian Science Foundation. A.E.S. acknowledges the Basis Foundation scholarship.

${ }^{1}$ I. I. Soloviev, N. V. Klenov, S. V. Bakurskiy, M. Y. Kupriyanov, A. L. Gudkov, and A. S. Sidorenko, Beilstein J. Nanotechnol. 8, 2689 (2017).

${ }^{2}$ R. Landauer, IBM J. Res. Dev. 5, 183 (1961).

${ }^{3}$ N. Takeuchi, Y. Yamanashi, and N. Yoshikawa, Sci. Rep. 4, 6354 (2014).

${ }^{4}$ Y. Harada and E. Goto, IEEE Trans. Magn. 27, 2863 (1991).

${ }^{5}$ M. Hidaka and L. A. Akers, Supercond. Sci. Technol. 4, 654 (1991).

${ }^{6}$ Y. Mizugaki, K. Nakajima, Y. Sawada, and T. Yamashita, Appl. Phys. Lett. 62, 762 (1993).

${ }^{7}$ Y. Mizugaki, K. Nakajima, Y. Sawada, and T. Yamashita, IEEE Trans. Appl. Supercond. 4, 1 (1994).

${ }^{8}$ P. Crotty, D. Schult, and K. Segall, Phys. Rev. E 82, 011914 (2010).

${ }^{9}$ Y. Yamanashi, K. Umeda, and N. Yoshikawa, IEEE Trans. Appl. Supercond. 23, 1701004 (2013).

${ }^{10}$ F. Chiarello, P. Carelli, M. G. Castellano, and G. Torrioli, Supercond. Sci. Technol. 26, 125009 (2013).

${ }^{11}$ J. M. Shainline, S. M. Buckley, R. P. Mirin, and S. W. Nam, Phys. Rev. Appl. 7, 034013 (2017).

${ }^{12}$ M. L. Schneider, C. A. Donnelly, S. E. Russek, B. Baek, M. R. Pufall, P. F. Hopkins, P. D. Dresselhaus, S. P. Benz, and W. H. Rippard, Sci. Adv. 4, e1701329 (2018).

${ }^{13}$ A. E. Schegolev, N. V. Klenov, I. I. Soloviev, and M. V. Tereshonok, Beilstein J. Nanotechnol. 7, 1397 (2016).
${ }^{14}$ N. V. Klenov, A. E. Schegolev, I. I. Soloviev, S. V. Bakurskiy, and M. V. Tereshonok, IEEE Trans. Appl. Supercond. 28, 1301006 (2018).

${ }^{15}$ S. K. Tolpygo, V. Bolkhovsky, D. E. Oates, R. Rastogi, S. Zarr, A. L. Day, T. J. Weir, A. Wynn, and L. M. Johnson, IEEE Trans. Appl. Supercond. 28, 1100212 (2018)

${ }^{16}$ K. K. Likharev, Int. J. Theor. Phys. 21, 311 (1982).

${ }^{17}$ V. V. Ryazanov, Phys. Usp. 42, 825 (1999).

${ }^{18}$ A. A. Golubov, M. Y. Kupriyanov, and E. Ilichev, Rev. Mod. Phys. 76, 411 (2004).

${ }^{19}$ S. V. Bakurskiy, N. V. Klenov, I. I. Soloviev, M. Y. Kupriyanov, and A. A. Golubov, Phys. Rev. B 88, 144519 (2013).

${ }^{20}$ S. V. Bakurskiy, N. V. Klenov, I. I. Soloviev, V. V. Bolginov, V. V. Ryazanov, I. V. Vernik, O. A. Mukhanov, M. Y. Kupriyanov, and A. A. Golubov, Appl. Phys. Lett. 102, 192603 (2013).

${ }^{21}$ S. V. Bakurskiy, N. V. Klenov, I. I. Soloviev, M. Y. Kupriyanov, and A. A. Golubov, Appl. Phys. Lett. 108, 042602 (2016).

${ }^{22}$ S. V. Bakurskiy, V. I. Filippov, V. I. Ruzhickiy, N. V. Klenov, I. I. Soloviev, M. Y. Kupriyanov, and A. A. Golubov, Phys. Rev. B 95, 094522 (2017).

${ }^{23}$ I. I. Soloviev, N. V. Klenov, A. E. Schegolev, S. V. Bakurskiy, and M. Y. Kupriyanov, Supercond. Sci. Technol. 29, 094005 (2016).

${ }^{24}$ L. V. Ginzburg, I. E. Batov, V. V. Bolginov, S. V. Egorov, V. I. Chichkov, A. E. Shchegolev, N. V. Klenov, I. I. Soloviev, S. V. Bakurskiy, and M. Y. Kupriyanov, JETP Lett. 107, 48 (2018).

${ }^{25}$ N. Takeuchi, Y. Yamanashi, and N. Yoshikawa, Supercond. Sci. Technol. 28, 015003 (2015).

${ }^{26}$ N. Takeuchi, D. Ozawa, Y. Yamanashi, and N. Yoshikawa, Supercond. Sci. Technol. 26, 035010 (2013).

${ }^{27}$ R. Caruso, D. Massarotti, V. V. Bolginov, A. Ben Hamida, L. N. Karelina, A. Miano, I. V. Vernik, F. Tafuri, V. V. Ryazanov, O. A. Mukhanov, and G. P. Pepe, J. Appl. Phys. 123, 133901 (2018).

${ }^{28}$ N. K. Katam, O. A. Mukhanov, and M. Pedram, IEEE Trans. Appl. Supercond. 28, $1300212(2018)$

${ }^{29}$ S. V. Bakurskiy, N. V. Klenov, I. I. Soloviev, N. G. Pugach, M. Y. Kupriyanov, and A. A. Golubov, Appl. Phys. Lett. 113, 082602 (2018).

${ }^{30}$ I. I. Soloviev, N. V. Klenov, S. V. Bakurskiy, V. V. Bolginov, V. V. Ryazanov, M. Y. Kupriyanov, and A. A. Golubov, Appl. Phys. Lett. 105, 242601 (2014). 